\title{
LA BIBLIOTERAPIA EN UN GRUPO DE MUJERES EN SITUACIÓN DE VIOLENCIA: FUNDAMENTOS CONCEPTUALES Y BALANCE DE INTERVENCIÓN
}

\section{Bibliotherapy in a Group of Women in Situations of Violence: Conceptual Foundations and Evaluation of an InTERVEntion}

\author{
Magda Ivette Moreno Morales* \\ Homero Ávila Landa**
}

DOI: http://dx.doi.org/10.29043/liminar.v19i2.850

\begin{abstract}
Resumen: En este artículo se presentan la fundamentación y los resultados obtenidos en una intervención educativo-terapéutica realizada con un grupo de veinte mujeres en situación de violencia. El objetivo consistió en favorecer el bienestar emocional por medio de la lectura de obras literarias seleccionadas para el tratamiento de temas afines a la situación de las participantes. Se utilizó el enfoque biblioterapéutico, el cual asume que la lectura tiene propiedades sanadoras. Los principales resultados muestran que la intervención produjo un incremento en el interés por continuar leyendo de forma individual y colectiva en espacios como el experimentado. Las participantes mostraron disposición para interpretar y relacionar los textos literarios con sus experiencias de vida.
\end{abstract}

Palabras clave: lectura, biblioterapia, violencia de género, promoción de la lectura, mujeres.

\begin{abstract}
This article discusses the foundation and results of an educational-therapeutic intervention carried out on a group of 20 women in situations of violence. The objective was to contribute to emotional well-being by reading selected literary works that deal with topics related to the situation of the participants. The library therapy approach is examined, which posits that reading literary works has healing properties. The main results indicate that the intervention encouraged greater interest in continuing to read individually and collectively in spaces such as the one experienced. Participants showed willingness to interpret and relate literary texts to their life experiences.
\end{abstract}

Key words: reading, bibliotherapy, gender violence, reading promotion, women.

\footnotetext{
* Magda Ivette Moreno Morales. Licenciada en Lengua y Literatura Hispánicas por la Universidad Veracruzana. Especialización en Promoción de la Lectura en la misma universidad. Asistente en el Centro de Estudios de la Cultura y la Comunicación de la Universidad Veracruzana, México. Temas de especialización: literatura de viajes, biblioterapia, promoción de la lectura. Correo electrónico: magdamorenomorales@ gmail.com. ORCID: https://orcid.org/0000-0001-7890-5764.

** Homero Ávila Landa. Doctor en Antropología Social. Investigador del Centro de Estudios de la Cultura y la Comunicación de la Universi-
}

dad Veracruzana, México. Temas de especialización: estudios culturales de la música popular, políticas culturales, participación ciudadana en el desarrollo cultural, estudios de las juventudes, identidades, culturas y tribus. Correo electrónico: havila@uv.mx. ORCID: https:/orcid. org/0000-0003-1187-2050.

Enviado a dictamen: 15 de julio de 2020

Aprobación: 15 de febrero de 2021 


\section{Introducción}

L a lectura puede generar estados de placer y satisfacción cuando se trata de una elección propia. En cambio, cuando es una actividad impuesta por la escuela o la familia, genera rechazo y desagrado para quienes se acercan a esta actividad (Garrido, 2014). No obstante, como actividad elegida desde la voluntad y la libertad individual, favorece el aprendizaje, enriquece el vocabulario, brinda diversas visiones de la vida y beneficia el razonamiento verbal y la experimentación de diversas emociones.

La literatura puede proveer un apoyo notable para afrontar los tiempos de angustia, para superar estados de ánimo o para cubrir necesidades de distracción en situaciones dominadas por la apremiante reproducción material y la vida cotidiana. El acto de lectura no solo mejora la capacidad para la comprensión de textos, la lectura también puede conducir a encontrar fuerza, algo inesperado que despierte la actividad psíquica, el pensamiento y la narración interior (Petit, 2009). El tipo de actividad lectora que interesa subrayar aquí es aquella que implica la dimensión subjetiva, es decir, que involucra el ámbito de las emociones, de las reflexiones articuladas y detonadas mientras se recorren los textos.

Para descubrir los beneficios no utilitarios de la lectura que respondan al bienestar emocional es necesario que esta actividad se convierta en un hábito. Por lo tanto, es preferible plantearla como recomendación por encima de asumirla como algo obligatorio. Sin embargo, el sistema educativo no ha sido capaz de coadyuvar en la formación de lectores, ya que generalmente en las escuelas se promueve la lectura como una herramienta para la apropiación del aprendizaje y no se enseña el camino para el disfrute de esta.

Para que alguien pueda experimentar la lectura y desarrollar el hábito lector, se requiere de motivaciones y sobre todo de acompañamiento (Argüelles, 2011; Garrido, 2014). Sin embargo, la mayoría de los proyectos de fomento a la lectura que buscan resarcir esta problemática enfocan sus iniciativas en el público infantil y juvenil, así como en el contexto escolar, dejando de lado otros grupos.
Las estadísticas muestran la baja proporción de adultos lectores. En el Módulo sobre lectura (MOLEC) se menciona que el promedio de libros leídos por la población de 18 años y más en el 2019 fue de 3.4 ejemplares (INEGI, 2020). Este problema también presenta sesgos de acuerdo con el género. Según datos del Instituto Nacional de Estadística y Geografía (INEGI) en México las mujeres leen menos que los hombres; en el MOLEC, las mujeres declararon leer un promedio de 3.2 ejemplares de libros al año a diferencia de los 3.7 registrados por los hombres (INEGI, 2020).

Aun cuando se conocen estas cifras, los programas de promoción de la lectura no suelen enfocar su atención en los grupos vulnerables o con necesidades particulares como los adultos mayores o las mujeres en situación de violencia. La violencia contra las mujeres en México es un problema grave que se refleja en las estadísticas, las cuales indican la urgencia de tomar acciones para afrontar esta problemática social desde diversos ámbitos y disciplinas. La educación puede ser una, ya que es una herramienta que puede brindar autonomía y autosuficiencia a las mujeres para salir de situaciones violentas. En este trabajo se propuso la lectura como ámbito de intervención en el marco de un programa provisto por una institución pública, el Instituto Municipal de las Mujeres de Xalapa (IMMX), que se encarga de proporcionar orientación jurídica y psicológica a mujeres en dichas circunstancias.

\section{La lectura, formas y usos}

La lectura por elección propia se denomina lectura recreativa; cuando conduce a un proceso cognoscitivo en el cual el lector o lectora es capaz de percibir la belleza generada por el texto, se conoce como lectura estética (Morales, 2019). Esta involucra la sensibilid ad del lector o lectora para distinguir aquello que le provoca una reacción sensitiva o emocional y, por ende, conlleva la experimentación de emociones y perspectivas de vida. Por el contrario, cuando se trata de una lectura para cumplir con una tarea, para resolver un problema u obtener información, de manera obligatoria o impuesta por la escuela o el trabajo, se conoce como lectura utilitaria 
(Jarvio y Ojeda, 2018). La lectura recreativa se realiza al margen de las actividades estrictamente obligatorias e impuestas por otras personas; se trata de una elección totalmente libre porque es el lector quien elige los textos de su preferencia, así también gestiona los tiempos que emplea para realizarla.

De acuerdo con Garrido (2012), la literatura es el tipo de lectura ideal para formar lectores autónomos, ya que los textos literarios tratan de la vida misma; de experiencias, sentimientos, conflictos y pasiones de los seres humanos, que son temas comunes a todos y con los que cualquier persona puede sentirse identificada.

Cuando no se siente como algo impuesto, una historia prestada o una frase puede muy pronto volverse parte de uno mismo y, al garantizar una distancia que protege, puede permitir evocar la propia historia en particular sus capítulos difíciles. Porque son sobre todo las páginas dolorosas de nuestra vida las que pueden ser leídas de manera indirecta (Petit, 2009:112).

Asentada la disposición o actitud positiva, es esperable que el acto de leer integre aprendizaje y experimentación; es también encuentro con uno mismo, con los otros y con lo otro, la posibilidad de conocer formas culturales, personales, colectivas e históricas o contemporáneas de la diversidad humana. Dispara resortes y sienta bases mediante los cuales los seres humanos nos identificamos con otros, con sus experiencias y situaciones. En palabras de Yunes (2005), la lectura de obras literarias estimula el reconocimiento del otro, lo que conduce a mirar más allá de los límites del "yo". La lectura nos interpela como seres humanos, como hombres o como mujeres, como seres que viven alegrías y frustraciones, que desean y sueñan. Leer nos revela nuestra pertenencia a una humanidad en constante transformación y reconfiguración. Por lo tanto, es posible hallar lecturas que quizá sin proponérselo orientan, abren, acompañan, fortalecen, dignifican.

Leer es una acción individual pero también una actividad colectiva: se lee lo que otros han vivido, lo que otros piensan, sienten, creen, imaginan, comparten, sugieren, etcétera. En palabras de Ouaknin, la lectura le ofrece al lector, por identificación y "cooperación textual", por apropiación y proyección, la posibilidad de descubrir una seguridad emocional, una alternativa para la realidad, una catarsis para los conflictos y la agresividad (2016:27). Por las razones antes mencionadas, la lectura semidirigida de obras literarias se ocupa como terapia, ya que hay un trabajo inconsciente del lector con sus propias emociones. De acuerdo con Petit (2015), el acto lector es terapéutico porque posibilita la reestructuración del pensamiento, lo que permite la visualización de múltiples posibilidades a una realidad, situación o problema.

\section{La apropiación lectora: la teoría transaccional de Rosenblatt y la teoría de esquemas}

Ambas teorías se articulan con el sentido terapéutico de leer ya que afirman que la lectura va más allá de descifrar un código de signos, y que más bien se trata de una transacción entre las ideas preconcebidas por el lector y el mundo narrado en la obra literaria. Ahora bien, la teoría de esquemas hace referencia a las concepciones que cualquier persona tiene sobre cualquier tema y aspecto de la vida cotidiana, con los cuales la lectura suele jugar; cuestionándolos o poniéndolos a prueba. Por eso, tal como señala Rosenblatt (1996), al leer estamos haciendo una autocrítica que nos lleva a repensar sentimientos y pensamientos. Entonces, en la medida en que los lectores analicen esos procesos y los interioricen, su lectura será mucho más completa comparada con aquella que se limita a observar aspectos superficiales del texto.

La aplicación de esta teoría como metodología en el fomento a la lectura se puede llevar a cabo mediante estrategias de lectura y escritura que busquen apropiarse del texto leído: dinámicas que inciten la reflexión mediante preguntas clave sobre la obra literaria leída con el objetivo de obtener respuestas oralizadas, que es una de las formas para apropiarse los textos, es decir, para internalizarlos y comprenderlos en grados más profundos de interpretación. También puede obtenerse mediante la escritura, la cual ayuda a ordenar el pensamiento, a elaborar una lectura crítica y a responder 
preguntas narratológicas. En ambos casos, la "respuesta expresada", permite la activación de ciertos aspectos del proceso que ocurren durante la lectura y la "interpretación expresada", contribuye en explicar y analizar la evocación sentida (Rosenblatt, 1996).

\section{La biblioterapia: concepto y aplicaciones}

Etimológicamente, biblioterapia viene de la unión de las palabras griegas biblio (libro) y terapia (cura o restablecimiento). Aunque existen varias definiciones del concepto, la mayoría de los especialistas coincide en que la biblioterapia es un programa que utiliza la lectura dirigida a partir de materiales seleccionados como coadyuvantes terapéuticos (Castro, 2016; Jalongo, 1983; Pérez de Rosell, 2011; Salaverti, 1999). Entonces, en el enfoque de la biblioterapia, la lectura tiene una función terapéutica que posibilita un bienestar emocional en los lectores o lectoras tras una selección previa de las obras basada en el contenido temático. Ouaknin (2016) afirma que el acto de la lectura en biblioterapia conlleva la interpretación de los significados adheridos al texto, y la interpretación es en sí misma una terapia ya que hace que el cerebro adquiera nuevas perspectivas, las cuales quedan contrastadas con las ideas preexistentes. Ette (2001) describe que se trata de un complejo juego interno que se establece por la oposición entre lo conocido y lo desconocido. Es decir, que cuando se leen obras literarias se balancea aquello que ya conocían los lectores o lectoras con lo que todavía no sabían y que adquieren tras la lectura del texto. Petit (2009) también afirma que la lectura es terapéutica, no obstante, su explicación se encuentra en las representaciones que ofrece la literatura sobre la vida, las cuales despiertan lo que está dormido o es ignorado en la mente del lector o lectora. Desde la perspectiva de esta autora, los libros pueden, en términos de su capacidad de transporte imaginario, mover al lector a generar nuevos pensamientos. En este sentido, leer puede expandir los horizontes y posibilitar nuevas perspectivas de pensamiento.

Así, interpretar y comprender una obra literaria puede conllevar la construcción de formas reflexivas de encuentro con uno mismo, pues la lectura comprendi- da e interpretada pasa a formar parte del lector, de su individualidad, de su situación, permitiendo maneras de reflexionar crítica y distanciadamente sobre afectaciones o emociones propias: "La lectura al suscitar la vida interior, desencadena un proceso terapéutico discreto" (Petit, 2009:113). Es a través de este ejercicio de autoconocimiento como la biblioterapia puede intervenir de modo favorable, buscando la mejora individual en contextos colectivos. Para Volpi (2018) la literatura posibilita experiencias diversas que sitúan al lector en escenarios de prueba y lo desvían de las formas tradicionales de resolver sus problemas, mecanismo que se concreta mediante las neuronas espejo.

Las obras que pueden producir bienestar emocional deben contener alguno de los seis componentes fundamentales de la biblioterapia que Caldin (2001) refiere y que a continuación se enlistan. El primero es la catarsis, que se entiende como un instrumento para pacificar las emociones, aunque cabe aclarar que al inicio estas pueden desencadenarse de manera intempestiva, en este caso a partir de un estímulo derivado de la lectura. El segundo se trata del humor, que alude a la capacidad de algunos textos para provocar risa, considerada también de carácter terapéutico. El tercero es la identificación, que señala la similitud que encuentra el lector entre su vida y la historia narrada en la obra literaria. El cuarto, la introyección, se trata de un proceso reflexivo en el cual el lector o lectora adopta pensamientos, cualidades o actitudes propias de los personajes, siendo estas positivas y pertinentes para su situación. El quinto es la proyección, definida como la transferencia a otros (personajes o historia de ficción) de nuestras ideas, sentimientos, intenciones, expectativas y deseos. El sexto es la introspección, que consiste en la autobservación mediante una actitud reflexiva sobre cualquier aspecto de la propia personalidad o conducta, de la propia conciencia o de los estados de ánimo.

Ello implica también la existencia de un mediador o biblioterapeuta que posea suficientes conocimientos literarios para identificar las potenciales propiedades terapéuticas de las lecturas, para que de este modo pueda proponer autores, textos y temas según la situación y los objetivos presentados. Asimismo, conlleva el uso de ha- 
bilidades didácticas por parte de este, de modo que leer tenga sentidos predeterminados, así como estrategias para abordar y manejar las necesidades y situaciones emocionales de cada persona o grupo de intervención

De acuerdo con lo anterior, tanto el perfil profesional del biblioterapeuta como el contexto en donde se realiza la intervención han dado como resultado una tipología de la biblioterapia. Naranjo Mora, Navarro y Zúñiga (2017), siguiendo a Daiva Janavičienè, distinguen dos tipos: la biblioterapia clínica y la biblioterapia educativa. La primera es aplicada por personal médico como un método adicional de tratamiento y se desarrolla en instituciones, hospitales y clínicas en las que se atiende a pacientes con enfermedades mentales, emocionales y físicas. La segunda puede ser aplicada por profesionales de los servicios sociales como bibliotecólogos, maestros, psicólogos, trabajadores sociales, promotores de lectura o literatos, y se trabaja en centros educativos, bibliotecas, centros juveniles e instituciones gubernamentales dedicadas a ofrecer servicios sociales. Este proyecto de intervención, con las adaptaciones del caso, se basa en la segunda clasificación de biblioterapia, es decir, la del tipo educativo.

\section{La violencia hacia las mujeres}

Antes de exponer el caso específico de intervención, se desarrolló el tema de la violencia hacia las mujeres, ya que las personas con las cuales se llevó a cabo la intervención eran mujeres que experimentaron algún tipo de violencia. Para adentrarnos en este tema, cabe hacer algunas precisiones al respecto. En el artículo uno de la Declaración sobre la Eliminación de la Violencia contra la Mujer de las Naciones Unidas se define la violencia contra las mujeres como:

Todo acto de violencia perpetuado contra el sexo femenino que tenga o pueda tener como resultado un daño o sufrimiento físico, sexual o psicológico para las mujeres, inclusive las amenazas de tales actos, la coacción o la privación arbitraria de libertad, tanto si se producen en la vida pública o privada (ONU, 1993).
La violencia hacia la mujer se presenta en todos los ámbitos y por parte de agresores diversos. En el artículo 6 de la Ley General de Acceso de las Mujeres a una Vida Libre de Violencia de 2018 se menciona que la violencia puede ser psicológica, física, sexual, patrimonial, económica o feminicida. Asimismo, hace referencia a los diferentes ámbitos en los cuales se desarrolla: familiar, laboral, escolar, comunitario e institucional.

La violencia hacia las mujeres es un fenómeno extendido y con complejas estructuras de pensamiento impuestas por la familia y por las instituciones políticas, educativas, religiosas y administrativas, así como en el medio laboral. Con estos mecanismos la sociedad impulsa a las personas a actuar de forma estereotípica según sea su género. Este reparto de papeles o roles ha originado un sistema de jerarquías que se conoce como patriarcado. En este, los hombres han predominado en todas las esferas de la vida, mientras que las mujeres reducen su labor al espacio doméstico de la familia. De acuerdo con el informe Desigualdades en México 2018 (Red de Estudios de Desigualdades, 2018), existen asimetrías contundentes en cuanto al género de las personas con estudios universitarios que se dedican a las labores del hogar, pues hay 80 veces más mujeres que hombres cuya ocupación principal son los quehaceres de su hogar. Cabe precisar que dedicarse a las labores del hogar no es una elección libre, ya que, si lo fuera, un número equivalente de varones con educación universitaria se ocuparía solo de labores domésticas (2018:58).

En la información captada por la Encuesta $\mathrm{Na}$ cional sobre la Dinámica de las Relaciones en los Hogares (ENDIREH) (INEGI, 2016) se observa que la violencia contra las mujeres es un problema de gran dimensión social ya que 30.7 millones de mexicanas han sido sujetas a actos violentos y discriminatorios en algún momento de su vida. La ciudad de Xalapa no es la excepción. La Línea Violeta que atiende el IMMX registró que en 2020 hubo un crecimiento de 40\% de los casos de violencia hacia la mujer, según datos proporcionados por la directora general del instituto en una entrevista realizada por el periódico el Quadratín Veracruz (Ortega, 2020). 


\section{Metodología de la intervención}

Se utilizó la metodología de investigación acción, que propone una acción reflexiva y crítica observando la realidad para transformarla y mejorarla (Latorre, 2005; Sequera 2016). La intervención educativo-terapéutica tuvo como objetivo favorecer el bienestar emocional de mujeres víctimas de violencia a través de fomentar la lectura de obras literarias. Se realizó bajo la modalidad de taller vivencial, en el cual las participantes fueron una parte activa de la intervención y no solo receptoras. Se diseñó un plan de trabajo con objetivos para cada sesión, estrategias y textos literarios con temas afines al grupo, actividades dinámicas, ejercicios de escritura creativa y de lectura en voz alta.

El plan de trabajo se presentó a la dirección del IMMX, donde propusieron algunas modificaciones de contenido y con quienes se acordaron horarios, visitas para conocer el contexto, así como los métodos de contención grupal y de intervención psicológica utilizados. Para el involucramiento en los talleres que imparte el IMMX, se consideraron conceptos del trabajo etnográfico, que plantea formas de interactuar en una comunidad determinada para conocer y registrar datos relacionados con su organización, saberes, intereses, etcétera (Peralta, 2009). Utilizando un diario de campo, se registró todo lo que se observó, escuchó y aprendió del grupo. Se puso una especial atención en las problemáticas, intereses y temas expresados, así como en actividades que disfrutaban las asistentes. Los resultados permitieron integrar al proyecto dinámicas de juego, uso de materiales reciclables y sesiones de dibujo, así como promover una actitud para escuchar, dialogar y empatizar en temas como las relaciones de pareja, el feminismo, la cultura, los estereotipos de género, experiencias dolorosas y de reflexión, así como de espacios para la convivencia.

El proyecto de intervención, diseñado como un taller de biblioterapia, se realizó del 14 de enero al 5 de marzo del 2020, los martes y jueves de 16:00 a 18:00 horas. Abarcó un total de dieciséis sesiones presenciales, cada sesión tuvo una duración de dos horas. Acudieron veinticuatro mujeres, de las cuales ocho eran externas al IMMX ya que la convocatoria fue abierta al público y difundida en redes sociales. Permanecieron hasta el final veinte asistentes, que fueron las que respondieron los cuestionarios, y las restantes explicaron su ausencia por motivos como dificultades en horarios y de traslado o cuestiones de salud, ya que una de ellas padecía cáncer, y una más manifestó que su interés se enfocaba al tema de la terapia clínica. El instituto nos proporcionó los datos de las usuarias del trimestre julio-septiembre de 2019; a ellas se les invitó directamente por teléfono y correo electrónico.

La selección de la cartografía lectora inicial se hizo con base en las consideraciones de pertinencia de la autora de este reporte. Además, se basó en las recomendaciones del libro Manual de remedios literarios. Cómo curarnos con los libros (Berthoud y Elderkin, 2017), que es un tratado a modo de diccionario de la A a la $Z$ y dividido en dolencias emocionales, donde se sugieren lecturas de acuerdo con las particularidades y vivencias del lector, basándose también en las propiedades biblioterapéuticas de las obras literarias aconsejadas por las autoras. Sin embargo, la selección se fue adecuando a lo largo de las sesiones hasta componer una cartografía final, la cual se construyó a partir de los resultados obtenidos en los cuestionarios aplicados, así como de las observaciones registradas en el diario de campo y las recomendaciones hechas por las psicólogas del IMMX. Debido a que no se encontraron investigaciones específicas sobre los efectos que cada una de las lecturas seleccionadas pudieran provocar, la propuesta de la cartografía se construyó partiendo también de la intuición y experiencia como lectora de la autora.

Asimismo, los resultados del diagnóstico ayudaron a la conformación de una cartografía lectora que abarcó cuentos, fragmentos de novelas y poemas cortos de autores nacionales e internacionales: María dos Prazeres de Gabriel García Márquez (2009), La ridícula idea de no volver a verte de Rosa Montero (2014), Una historia sobre peces de Ajahn Chah (2005), El lobo estepario de Herman Hesse (2008), El horla de Guy de Maupassant (2007), Seapor Dios y venga más de Laura Esquivel (2010), Gotas de Edgar Keret (2009), La carta de Amparo Dávila (2016), No te rindas y ¿Cómo hacerte saber que siempre hay tiempo? de 
Mario Benedetti (2020), La casa de Asterión de Jorge Luis Borges (2011), Consejos para la mujer fuerte de Gioconda Belli (2019), Mujeres de ojos grandes de Ángeles Mastretta (2012), La luna de Jaime Sabines (1973) y Poema a Margarita de Rubén Darío (2020).

Se utilizaron cuentos infantiles ilustrados, elegidos por sus contenidos temáticos afines a las necesidades e intereses expresados por el grupo de mujeres en el segundo cuestionario aplicado. Por ejemplo: El pájaro del alma de Mijal Snunit (2009), La gran fábrica de palabras de Agnès Lestrade (2013), Todos somos diferentes de Alicia Molina (2015), Con la cabeza en otraparte de André Bouchard (2013), Lucy y el monstruo de Ricardo Bernal (2014), La ardilla miedosa de Mélanie Watt (2007), Yo voy conmigo de Raquel Reguera Díaz (2015), El cazo de Lorenzo de Isabelle Carrier (2013), El corazón y la botella de Oliver Jeffers (2010), El libro de los cerdos de Anthony Browne (1991) y El abrazo de David Grossman (2019). Para la sesión 6 se utilizaron textos relacionados con la biblioterapia.

El tema de cada sesión se comentaba y se mostraba de forma implícita o explícita en los textos y ejercicios. Los temas abordados fueron sugeridos por las dos psicólogas: ${ }^{1}$ 1) El conocimiento intuitivo de nuestro ser emocional; 2) Los recursos interiores para afrontar la vida y la importancia de las palabras; 3) La construcción de la identidad y el amor propio; 4) Afrontar los miedos y otros monstruos; 5) Los laberintos de la mente; 6) ¿Qué es biblioterapia y cómo se aplica?; 7) La naturaleza y la ecología; 8) Reconstruyendo mi autoestima; 9) Lectura de emociones; 10) El apego y el desapego en los vínculos afectivos; 11) Empoderamiento femenino; 12) Depresión, tristeza y melancolía; 13) Nuevas perspectivas de la soledad; 14) La conciencia sobre la finitud del ser humano; 15) Escritura creativa y poesía, y 16) Reflexiones y clausura del taller (ver Figura 1).

La dinámica de las sesiones, a excepción de la sesión 16 que fue la clausura, la sesión 6 que se basó en la teoría de la biblioterapia y la 7 en la que se proyectó un documental, siguieron la siguiente estructura: dos lecturas en voz alta, una grupal, en la cual leían de forma voluntaria las participantes y la otra dirigida por la coordinadora del taller, generalmente después de la primera activi- dad dinámica. Asimismo, se realizaron dos actividades prácticas por día: una dinámica de integración grupal al inicio de la sesión y otra de expresión escrita, después de las dos lecturas en voz alta, al final de la sesión.

Para evaluar si la sesión cumplió el objetivo planteado se diseñó un esquema que integró la elaboración y aplicación de dos cuestionarios diagnósticos para conocer la edad, el estado civil, la ocupación profesional, así como las preferencias y prácticas de lectura, la situación emocional y en específico la presencia de los componentes que menciona Caldin (2001) como la identificación, la introyección, la proyección, la introspección, etcétera.

El primer cuestionario permitió conocer las características personales de las asistentes al taller. El segundo cuestionario fue aplicado a la mitad del total de sesiones. Este brindó información sobre actividades cotidianas, sobre pasatiempos y sobre su acercamiento a la lectura; también hubo un apartado en el cual las participantes propusieran temas a tratar para las siguientes sesiones.

Se utilizó un diario de campo donde se registró lo siguiente: número de asistentes, reacciones, actitudes, objetivos, lecturas utilizadas, así como las evidencias y reflexiones más destacadas. Esta información se procesó en una hoja de cálculo en el programa Excel mediante tablas y gráficos de barras. Finalmente se realizaron entrevistas semiestructuradas para evaluar la experiencia obtenida, así como el impacto resultante de la aplicación de la biblioterapia. La entrevista fue voluntaria y se hizo mediante preguntas semiestructuradas; solamente seis del total de las participantes aceptaron realizarla.

\section{Resultados}

De acuerdo con los datos recabados, el rango de edad oscila entre los 21 y 70 años. En este sentido, el grupo conformado fue muy diverso en cuanto a las edades y experiencias de vida, lo cual configuró cierta complejidad y representatividad social, y una riqueza de trayectorias, vivencias e ideas, las cuales terminaron animando el taller. No obstante, solo el 5\% (una) de las asistentes eran adultas mayores, con un rango de edad entre 61 y 70 años. En número seguían las mujeres de entre 41 y 
50 años, que conformaban el 15\% (tres) del total. Había un 25\% (cinco) de mujeres entre los 51 y los 60 años, la población mayoritaria se hallaba en el rango de 31 a 40 años, que sumaban el 30\% (seis) de las mujeres, y el resto de las asistentes, es decir, un 25\% (cinco), eran mujeres de 21 a 30 años.

De las veinte encuestadas, trece lograron terminar una licenciatura en diferentes periodos, cuatro culminaron el bachillerato, dos la secundaria y una indicó que únicamente tuvo la oportunidad de concluir la primaria. Por lo tanto, los perfiles fueron bastante diversos, así como las habilidades de cada una. No obstante, esto no disminuyó su nivel de participación y disponibilidad para aprender o apoyar al resto del grupo. Respecto a sus ocupaciones laborales, del total de asistentes, nueve tenían autoempleo, tres eran estudiantes, cinco empleadas y tres se dedicaban a las labores del hogar.

Desde la sesión número 3 las participantes mostraron interés por conocer la biblioterapia como herramienta para tratar problemáticas emocionales. En el diario de campo se registró que al finalizar la sesión 3 se acercaron algunas de ellas para pedir recomendaciones de libros que fueran adecuados para ciertas circunstancias relacionadas con familiares o amigos. Por mencionar algunos ejemplos, solicitaron un libro para una hija adolescente a la que no le gustaba su cuerpo, otro para una prima que había tenido intentos de suicidio y otro más para una amiga que vivía un duelo amoroso debido a su divorcio.

La planeación de la sesión 6 surgió a raíz del interés y la curiosidad del grupo por conocer el funcionamiento y los beneficios de la biblioterapia. Consistió en proporcionarles teoría y bibliografía sobre el tema, así como una lista de libros que ellas podrían ocupar en diferentes situaciones. Esta sesión no estaba contemplada, se llevó a cabo por petición de las asistentes. Los datos recopilados en el segundo cuestionario también confirman el interés experimentado por las veinte mujeres del taller a lo largo de las sesiones, ya que, aunque quince de las participantes no tenían ninguna experiencia en círculos o talleres de lectura, al finalizar la intervención el cien por ciento de ellas expresó que les gustaría participar en otro taller de biblioterapia. Otra forma en la cual se evaluó el interés del grupo fue mediante el registro de asistencias a lo largo de las sesiones, que se mantuvo en un promedio de quince.

Las mujeres participantes en la intervención educativo-terapéutica tenían expectativas concretas del taller Arte(sanas) de la Palabra: de las veinte participantes, seis esperaban sanar emociones, cinco afirmaron que buscaban aprender, compartir y hacer amigas, tres querían adquirir conocimientos terapéuticos, otras tres deseaban ampliar sus conocimientos literarios, una participante buscaba adquirir el hábito lector, una anhelaba capacitarse para ayudar a otras personas, y una última afirmó que quería tener un mejor conocimiento de sí misma.

Por otra parte, de las veinte participantes diecinueve expresaron que desconocían el significado del concepto biblioterapia y que tampoco habían participado en un taller de esta naturaleza.

Tras la lectura de varias obras literarias, entre las favoritas destacaron los cuentos: El pájaro del alma de Mijal Snunit, Todos somos diferentes de Alicia Molina, La ardilla miedosa de Mélanie Watt y La casa de Asterión de Jorge Luis Borges. En ellos destacan dos aspectos: un lenguaje sencillo pero abundante en metáforas atractivas y fáciles de comprender para un lector primerizo, así como el tratamiento directo de temas afines a las necesidades del grupo (alusivos a la autoestima, el amor propio, el duelo, la inclusión, la expresión de emociones, etcétera). Los cuentos infantiles contenían ilustraciones llamativas y menos páginas, por lo tanto fueron más comentados y retenidos.

Con base en las cuatro lecturas favoritas de las participantes, se les preguntó lo que experimentaron con cada una de ellas en relación con los seis componentes fundamentales según Caldin (2001) que se les explicaron y presentaron en la sesión 6 de biblioterapia. Esta evaluación fue realizada a partir del diario de campo y de las evidencias obtenidas a través de los ejercicios prácticos de escritura correspondientes a las lecturas mencionadas: se analizaron estas evidencias y se identificaron los componentes fundamentales experimentados por cada una de las participantes. 
Cabe aclarar que no tuvimos como objetivo explicar cómo una obra literaria detona determinados componentes, sino que nos enfocamos en seleccionar obras literarias que en potencia pudiesen presentar estos efectos en las lectoras participantes de este taller. Por mencionar un ejemplo, en un principio se adjudicó al cuento La ardilla miedosa el componente de humor ya que se pensó que podría producir un efecto cómico pues el autor juega a ridiculizar el miedo a las alturas y a los riesgos a través del personaje principal, que además posee una exagerada previsión por el futuro. No obstante, la recepción de esta obra no tuvo el efecto de risa esperado, sino que provocó ensimismamiento y reflexión. De forma similar ocurrió con la introyección, la cual se pensó que podría ser experimentada en la lectura de Todos somos diferentes ya que en esta historia la protagonista nace con una cola de quetzal que la hace sentirse excluida, pero después de escuchar las palabras de su abuela, consigue amarla y utilizarla con creatividad. Sin embargo, esta lectura no indujo a que las lectoras adoptaran pensamientos o actitudes positivas del personaje, como sucede cuando se presenta la introyección, sino que recordaron situaciones en las que no se aceptaron a sí mismas o sintieron el rechazo de otros (ver Figura 2).

En la sesión uno, a la cual acudieron dieciséis participantes, la lectura evaluada con esos componentes fue Elpájaro del alma. De las asistentes, seis se sintieron identificadas con la narración del texto, otras seis manifestaron una proyección de sus emociones y pensamientos en el protagonista, es decir, en el pájaro del alma, tres sintieron catarsis con la lectura y una introspección. En la sesión 3 se hizo la lectura de Todos somos diferentes; a esta sesión acudieron diecisiete mujeres, de las cuales ocho se sintieron identificadas con la historia, a seis de ellas la lectura les provocó introspección en torno a sus vivencias y pensamientos, dos pasaron por la catarsis y una por la proyección. En la sesión 4, a la cual asistieron quince mujeres, la lectura evaluada fue La ardilla miedosa. De las participantes, diez de ellas experimentaron la identificación con la lectura, tres introspección y dos proyección. En la sesión 5, a la cual asistieron diecinueve mujeres, se leyó La casa de Asterión; en este caso, siete ex- perimentaron proyección, cinco introspección, cuatro de ellas identificación y dos catarsis.

Aunque solo las cuatro lecturas favoritas fueron evaluadas respecto a la experimentación de los componentes fundamentales de la biblioterapia, eso no implicó que los componentes estuviesen completamente ausentes en otros textos. También en la entrevista se registró la presencia de uno de los componentes fundamentales de la biblioterapia.

Promotora de lectura: ¿Cuál fue la experiencia más significativa que tuviste en el taller?

Participante: Pues la experiencia más significativa fue una sesión donde salió el tema del acoso, porque era una situación actual en mi vida, y relevante para mí, por haberla vivido. Pues pasaron muchas cosas cuando viví eso. Durante esa sesión sentí todo muy intenso, como que ese día procesé la información que a lo mejor en días anteriores no había tenido la reflexión de lo que había pasado, o sea, sí me sentí muy vulnerable, pero fíjate que me sirvió muchísimo la actividad de reescribir el final del cuento que nos pusiste, porque fue una manera como de escribir mi propio final, de mi propia historia. O sea, primero tuve sentimientos muy abrumadores, porque tocar el tema fue volver a tocar la herida, porque vi la situación sin filtros, pero en el segundo tiempo, cuando escribimos el final de la historia, fue un alivio para mí, una forma de zurcir la herida (AM, 27, secretaria).

Primero, la participante describe haber experimentado "sentimientos muy abrumadores" cuando se analizó y comentó el cuento Lucyy el monstruo, los cuales le crearon dolor, pues como ella dice, "vio la situación sin filtros, fue como volver a tocar la herida". No, obstante, cuando realizó la actividad de escritura creativa, que trataba de escribir un final diferente al cuento, la participante AM describió que fue como "zurcir la herida, un alivio". Esto confirma lo escrito por Caldin (2001) sobre la catarsis como una de las propiedades de las obras biblioterapéuticas, en las cuales el lector pasa por un proceso de liberación de las emociones, en el sentido de 
la catarsis aristotélica. Para Ouaknin (2016) la catarsis se produce por esa alquimia subjetiva de la literatura que consiste en transformar en placer la pena inherente a las emociones como la piedad o el terror. Es decir, que la turbación sentida en la experiencia real de acoso de la participante AM, cuando es experimentada en la lectura de una representación artística, en este caso el cuento citado, deja de ser una emoción violenta como la de la vida, pues está desprovista de su fuerza nociva. Por lo tanto, da origen a una especie de alivio.

Siguiendo la propuesta y la perspectiva de lectura de Rosenblatt (1996), se analizaron las respuestas expresadas en la entrevista para conocer qué tanto se apropiaron de los textos, es decir, si los contenidos fueron significativos, si pudieron internalizarlos y comprenderlos en profundidad o relacionarlos con sus experiencias de vida. Asimismo, con estas mismas respuestas se describieron qué actividades y obras literarias incitaron el diálogo y la reflexión.

\begin{abstract}
Promotora de lectura: ¿Qué autores y textos representaron para ti un auxilio psicológico y emocional? Participante: Todos los textos del taller fueron bastante enriquecedores, sin embargo, los que más recuerdo son la lectura de Mujeres de ojos grandes de Ángeles Mastretta y iSeapor Dios y venga más! de Laura Esquivel. Los recuerdo perfectamente porque fue impresionante para mí descubrir cómo tenemos tan arraigado la cultura del machismo, cómo la normalizamos, pero cada día somos más mujeres las que nos damos cuenta de que no es lo correcto, que no está bien, y empezamos a cuestionar las prácticas que tenemos con nosotras mismas y para con el resto de las mujeres (DG, 23 años, estudiante universitaria).
\end{abstract}

La participante DG mencionó los textos que fueron más provechosos para ella. En ambos casos, las protagonistas son mujeres que dan un giro a su vida porque deciden por sí mismas sin reproducir los roles de género asignados a las mujeres. No obstante, de acuerdo con las notas del diario de campo, estas mismas lecturas también generaron inquietud y silencio en la mayoría de las participantes del grupo, excepto en las más jóvenes
(DG 23 años, DC 26 años, AM 27 años, KM 23 años y LM 29 años), quienes estuvieron a cargo de la discusión grupal y hablaron sobre temas como los roles de género, el machismo, el feminismo y el heteropatriarcado. Entonces, los textos que contienen tópicos de la lucha de las mujeres, si bien generaron reflexión y diálogos, en estos solo participaron algunas usuarias: las que sí tenían interés en la lectura o algunos conocimientos previos que pudieron utilizar o asociar para dar contexto y significado a las lecturas.

En cambio, tras la lectura de Todos somos diferentes se pudo notar mucho entusiasmo en la mayoría de las asistentes para explicar y comprender la lectura; asimismo, hubo mucha participación para relacionar el texto con sus experiencias de vida y expusieron diferentes casos en los cuales ellas fueron discriminadas; dos señoras compartieron su experiencia por ser estudiantes adultas (CJ 52 años y MH 56 años), una de ellas sí logró concluir sus estudios y la otra señora platicó entre lágrimas que fue un proceso duro que no pudo sobrellevar debido a las exigencias requeridas sobre todo en el uso de la tecnología; se sentía excluida y no comprendida por sus compañeros y maestros.

En el cuestionario realizado también se les preguntó si habían compartido los textos y los aprendizajes del taller con otras personas, ya que es un indicador de qué tan implicadas e interesadas se sintieron las participantes en las sesiones porque un conocimiento es significativo cuando se busca compartirlo con otras personas, con lo que se refuerza el aprendizaje en la persona que lo posee.

Cuando se les preguntó si compartieron los textos y los aprendizajes del taller: diecisiete participantes respondieron que sí, contra tres que respondieron que no. Es decir, que el 85\% del grupo decidió compartir lo experimentado. Se les preguntó también con quiénes compartieron los textos: doce de ellas manifestaron que con su familia, tres con sus amigas, dos participantes afirmaron que con sus vecinos, dos con sus alumnos a los que les impartían clases y una con sus compañeros de trabajo.

Con respecto a los aprendizajes obtenidos: dos dijeron que aprendieron sobre el autocuidado de su 
mundo interior; otras dos hicieron referencia al valor de las palabras y a la importancia de analizar textos; tres sobre conocer sus habilidades y recursos emocionales para afrontar su vida; seis asimilaron la importancia de vincular las lecturas con las experiencias personales; cuatro aprendieron a leer cuentos infantiles desde la óptica terapéutica, y tres a vincular la literatura con la terapia.

En el segundo cuestionario se les pidió que calificaran qué tan libres se sentían para compartir sus pensamientos, vivencias y emociones con sus compañeras: en otras palabras, si el ambiente creado en el taller les facilitaba abrirse emocionalmente. La escala para calificarlo fue del 1 al 10, tomando el 10 como la máxima calificación positiva, en la que se sienten totalmente libres y cómodas para expresarse, mientras el 1 haría referencia a la mínima calificación negativa, aludiendo a un ambiente de inseguridad e incomodidad. De las veinte mujeres, solamente una participante lo calificó con 7 , seis participantes lo calificaron con 8 , cinco lo calificaron con 9 y ocho con 10, siendo el promedio de 8.9. De acuerdo con lo anterior, se construyó que el ambiente era seguro y respetuoso, pues las participantes que calificaron el ambiente con 9 y 10 sumaban el $70 \%$ del total.

El taller de lectura también favoreció el conocimiento intrapersonal ya que propició emociones positivas en las participantes; estos elementos se manifestaron a través de los ejercicios de escritura y las dinámicas de lectura. A continuación se presenta un testimonio al respecto.

Promotora de lectura: De acuerdo con tus vivencias en el taller de biblioterapia, iconsideras que la lectura de obras literarias puede coadyuvar en tu bienestar emocional?

Participante: Sí, porque los libros nos muestran situaciones y vivencias que abren otras perspectivas y que podemos tomar como referencia y tener más posibilidades de respuestas ante un hecho específico. El taller me ayudó a valorar más la lectura como fuente de conocimientos y como medio para canalizar estrés y emociones negativas. El disfrutar de una lectura co- mentada y la convivencia con el grupo de compañeras lo hizo más enriquecedor por las aportaciones de cada una desde sus experiencias personales (MH, 56 años, labores del hogar).

En las respuestas proporcionadas en la entrevista, manifestaron que a partir de las reflexiones derivadas de las sesiones contaban con más elementos para hacer frente a determinadas situaciones o para evaluar otras (pertenecientes al pasado) con una perspectiva más amplia. Del total de las asistentes, únicamente dos afirmaron sentirse melancólicas y tristes durante las sesiones, principalmente porque se trataba de emociones con las que ya cargaban antes de integrarse al taller. Por otro lado, diez participantes manifestaron sentirse contentas y reflexivas, siete se describieron como interesadas y motivadas, y solo una de las veinte afirmó haberse sentido distraída y aburrida durante la mayor parte de las sesiones, lo que argumentó porque los comentarios de sus compañeras eran muy largos.

\section{Conclusiones}

La intervención realizada permitió descubrir un camino nuevo respecto a los usos y beneficios de la lectura de obras literarias. Cabe aclarar que la intervención no buscó transformar las condiciones de violencia que experimentaban las participantes, tampoco medir o analizar tales condiciones, sino ofrecer una propuesta de actividad como adyuvante al proceso terapéutico que ya recibían en el IMMX.

Dialogar sobre las experiencias y aprendizajes adquiridos en el taller posibilitó en las participantes: reforzar lo aprendido, ampliar su comprensión y también coadyuvar en la creación de nuevos espacios para reflexionar sobre temas como los tratados en las sesiones con familiares, vecinos y amigas o amigos. Este aspecto fue importante para la evaluación del impacto del taller, en el que uno de los rubros de evaluación era la iniciativa de las participantes por compartir o no sus vivencias y textos con otras personas.

Los resultados del segundo cuestionario aplicado muestran que la intervención produjo en las participan- 
tes un interés por continuar leyendo de forma individual y colectiva en espacios como el experimentado y que las lecturas seleccionadas condujeron al diálogo y la reflexión. Asimismo, las integrantes del taller mostraron disposición para interpretar y relacionar los textos literarios con sus experiencias de vida, o bien para ampliar y enriquecer su realidad por medio de la lectura. La mayoría de ellas calificó el ambiente del taller como propicio para expresar sus emociones y pensamientos.

La biblioterapia como alternativa se prolongó más allá del taller, de la institución y del programa mismo, porque el interés activo de las participantes se desplazó a una plataforma comunicacional actual digital, ya que el grupo continuaba activo por medio de WhatsApp después de diez meses de haber finalizado la intervención. Este espacio virtual permite que los diálogos entablados en el taller se sigan enriqueciendo, que las participantes tengan la oportunidad de compartir otros textos que les resulten pertinentes, así como que puedan hacerse recomendaciones de obras literarias que hayan leído recientemente.

Asimismo, uno de los puntos fuertes del taller de biblioterapia fue el ambiente creado, ya que se conformó una colectividad en torno a un interés común, la lectura de obras literarias, el cual fue descrito como un soporte de cariño y empatía, pues permitió a las mujeres sentirse confortadas, libres y cómodas para expresarse.

Con base en el registro llevado a cabo, y en el análisis de este y de los avances de cada participante, puede afirmarse que la intervención biblioterapéutica favoreció los estados emocionales de la mayoría de las participantes. El testimonio de la participante AM previamente presentado es un ejemplo del efecto catártico de una obra literaria en el lector que se siente identificado con la narrativa. Los análisis de los textos literarios permitieron a las mujeres hacer ejercicios de reflexión y autoconsciencia a través de los componentes fundamentales de la biblioterapia que se presentaron durante las lecturas.

Por lo anterior, se recomienda la puesta en práctica de proyectos de biblioterapia a largo plazo, así como la formación profesional de biblioterapeutas habilitados en diferentes temas de carácter psicológico y social, con una constante actualización literaria y pedagógica para la facilitación de los talleres.

Finalmente, se señala la conveniencia de incorporar talleres de este tipo en las instituciones públicas y espacios sociales que atienden a personas con necesidades específicas que requieren acompañamiento, principalmente grupos vulnerables. En el caso específico de la propuesta de biblioterapia aquí mostrada, se considera que puede ser una actividad coadyuvante a un proceso terapéutico, porque partiendo de ejercicios de lectura es posible alcanzar formas de autorreflexión grupal e individual.

\section{Notas}

${ }^{1}$ Las dos psicólogas fueron asignadas por el IMMX. Las funciones de las psicólogas fueron de contención emocional del grupo, intervención en caso de crisis y asesoría externa dentro del contexto del IMMX dirigida a las asistentes que en ese momento no estaban llevando un proceso terapéutico o no eran usuarias del Instituto. Las psicólogas se turnaban para la asistencia al taller, una acudía todos los martes y la otra todos los jueves.

\section{Referencias}

Argüelles, Juan Domingo (2011). Escribir y leer con los niños, los adolescentes y los jóvenes. México: Océano.

Berthoud, Ella y Susan Elderkin (2017). Manual de remedios literarios. Cómo curarnos con los libros. Madrid: Siruela.

Caldin, Clarice Fortkamp (2001). "La literatura como función terapéutica”. En Encontros Bibli: Revista Electrónica de Biblioteconomía y Ciencias de la Información, 12(6) 22-44. Disponible en https://periodicos.ufsc.br/index.php/eb/article/view/1518-2924.2001v6n12p32/ (consultado el 26 de octubre de 2019).

Castro Viguera, Yenifer (2016). "La biblioterapia y la selección de fuentes de información: un ámbito de actuación para los profesionales de la bibliotecología y la ciencia de la información". En Bibliotecas. Anales de Investigación, 13(1), 82-95. Disponible en http://revistas.bnjm.cu/index.php/anales/article/view/3666 (consultado el 10 de septiembre de 2019). 
Ette, Ottmar (2001). Literatura de viaje: de Humboldt a Baudrillard. México: UNAM.

Garrido, Felipe (2012). Manual del buen promotor: Una guía para promover la lectura y la escritura. México: Educal.

Garrido, Felipe (2014). El buen lector se hace, no nace: reflexiones sobre lecturay formación de lectores. México: Paidós.

INEGI (Instituto Nacional de Estadística y Geografía de México) (2020). Módulo sobre lectura (MOLEC). Disponible en https://www.inegi.org.mx/contenidos/programas/ molec/doc/resultados_molec_feb20.pdf (consultado el 20 de agosto de 2020).

INEGI (Instituto Nacional de Estadística y Geografía de México) (2016). "Encuesta Nacional sobre la Dinámica de las Relaciones en los Hogares (ENDIREH)". Disponible en https:/www.inegi.org.mx/programas/ endireh/2016/ (consultado el 10 de agosto de 2020).

Jarvio Fernández, Olivia y Mario Miguel Ojeda Ramírez (2018). "Lalecturanoutilitariaenlauniversidadenlaeradigital. Un análisis multivariante que ubica el texto impreso en la lectura de literatura". En Palabra Clave(La Plata), 7(2), 1-16. Disponible en https://doi.org/10.24215/18539912e051 (consultado el 8 de agosto de 2020).

Jalongo, Mary Renck (1983). "Bibliotherapy: Literature to Promote Socioemotional Growth". En The Reading Teacher, 36, 796-803. Disponible en https://eric. ed.gov/?id=EJ276258 (consultado el 8 de agosto de 2020).

Latorre Beltrán, Antonio (2005). Lainvestigación-acción. Conocer y cambiar la práctica educativa. Barcelona: Editorial Graó.

Morales Sánchez, M. Isabel (2019). "La lógica de la lectura: de la lectura estética o el arte de leer con sentido". En Elsa Margarita Ramírez Leyva (coord.), De la lectura académica a la lectura estética. México: UNAM.

Naranjo Mora, Kimberly, Gloriela Navarro Araya y Tatiana Zúñiga Seravalli (2017). "La biblioterapia como herramienta de ayuda aplicada en la biblioteca escolar: estudios de caso". En E-Ciencias de la Información, 7(2), 1-26. Disponible en https://revistas.ucr.ac.cr/index.php/ eciencias/article/view/29259 (consultado el 5 de septiembre de 2019).

ONU (Organización de las Naciones Unidas) (1993). "Declaración sobre la Eliminación de la Violencia contra la Mujer. Resolución de la Asamblea General 48/104, de 20 de diciembre de 1993". Disponible en http://
servindi.org/pdf/DecEliminacionViolenciaMujer.pdf (consultado el 10 de septiembre de 2019).

Ouaknin, Marc-Alain (2016). Biblioterapia: leer es sanar. México: Océano.

Ortega, Samantha (2020). "Incrementa en Xalapa violencia contra mujeres". En Quadratín Veracruz, 13 de abril. Disponible en https:/veracruz.quadratin.com.mx/incrementa-en-xalapa-violencia-contra-mujeres-immx/ (consultado el 20 de abril de 2020).

Peralta, Claudina (2009). "Etnografía y métodos etnográficos". En Análisis. Revista Colombiana de Humanidades, (74), 33-52. Disponible en https://www.redalyc.org/ articulo.oa?id=5155/515551760003 (consultado el 17 de mayo de 2020).

Pérez de Rosell, Milagros (2011). "Literapia como práctica terapéutica de los estudiantes de Autodesarrollo del DCS en el Hospital Universitario de Pediatría 'Agustín Zubillaga' de Barquismeto. Una experiencia educativa”. En Boletín Médico de Posgrado, 28, 109-117. Disponible en https://core.ac.uk/download/pdf/71504457.pdf (consultado el 18 de mayo de 2020)

Petit, Michèle (2009). El arte de la lectura en tiempos de crisis. Madrid: Océano.

Petit, Michèle (2015). Leer el mundo: experiencias actuales de transmisión cultural. Buenos Aires: Fondo de Cultura Económica de Argentina.

Rosenblatt, Louise (1996). "La teoría transaccional de la lectura y la escritura". En Textos en Contexto, (1), 1-39.

Red de Estudios sobre Desigualdades (2018). Desigualdades en México 2018. México: El Colegio de México. Disponible en https://desigualdades.colmex.mx/informe-desigualdades-2018.pdf (consultado el 20 de junio del 2020).

Salaverti Pitarch, Pepa (1999). "La biblioteca de usuarios del Hospital Universitario La Fe de Valencia”. En Métodos de Información, 6, 74-76.

Sequera, María (2016). “Investigación-acción: un método de investigación educativa para la sociedad actual”. En RevistaArjé, 18(10), 223-229. Disponible en http://arje.bc.uc. edu.ve/arj18/art23.pdf

Volpi, Jorge (2018). Leer la mente. El cerebro y el arte de la ficción. México: Alfaguara.

Yunes, Eliana (2005). La presencia del otro en la intimidad del yo: aprendiendo con la lectura. México: CONACULTA. 
Figura 1. Tabla ejemplo de la configuración de la cartografía literaria

\section{Sesión 8.6 de febrero de 2020}

Reconstruyendo mi autoestima

Objetivo de la sesión:

Que las usuarias se hagan conscientes de los factores (sociales, culturales, familiares o de la percepción de otros) que han influido en la construcción de su propia estima a lo largo de su vida a través del texto seleccionado para la sesión.

Lecturas seleccionadas:

Lectura central: Reguera Díaz, R. (2015). Yo voy conmigo. Thule Ediciones.

Lectura de cierre: Riso, W. (2016). Enamórate de ti mismo: el valor imprescindible de la autoestima. Editorial Océano.

Dinámica de integración: las usuarias forman un círculo. Se les otorgan unas lunetas de chocolate al azar. Por cada color que tengan deben relatar o decir lo que les corresponde:

Blanca: Un obstáculo o reto superado.

Rosa: Un defecto que me han apuntado otras personas.

Naranja: Una cualidad que percibo en mí

Actividad recreativa: creación de autorretrato. Se reparten materiales diversos para las usuarias elaboren un autorretrato de la forma más creativa posible. Las usuarias deberán escribir alrededor de su autorretrato algunos adjetivos que las describan. La actividad es a contrarreloj. Se les brindará un minuto para finalizar esta acción, ya que se busca una escritura espontánea. Cuando finalicen, comparten su representación al grupo. Las mediadoras harán algunas preguntas para guiarlas a la introspección sobre lo esquemas bajo los cuales construyen su autoestima.

Figura 2. Participantes que experimentaron alguno de los componentes fundamentales de la biblioterapia

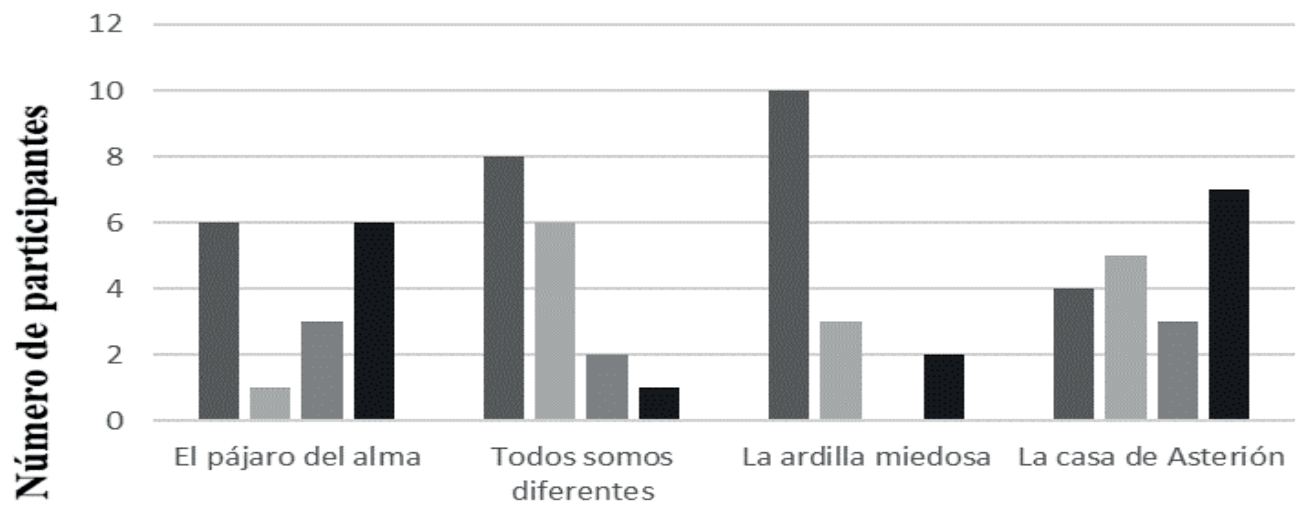

\section{Lecturas utilizadas}

- identificación Introspección a Catarsis a Proyección 